\title{
Volcanic Plumes: Impacts on the Atmosphere and Insights into Volcanic Processes
}

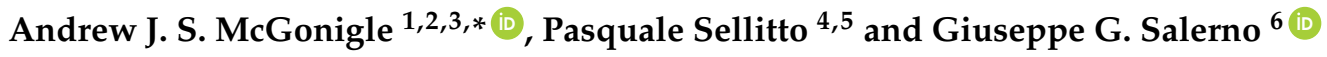 \\ 1 Department of Geography, University of Sheffield, Winter Street, Sheffield S10 2TN, UK \\ 2 School of Geosciences, The University of Sydney, Sydney NSW2006, Australia \\ 3 Istituto Nazionale di Geofisica e Vulcanologia, Sezione di Palermo, Via Ugo La Malfa 153, \\ 90146 Palermo, Italy \\ 4 Laboratoire de Météorologie Dynamique, Institut Pierre Simon Laplace, École Normale Supérieure, PSL \\ Research University, École Polytechnique, Université Paris-Saclay, Sorbonne Université, UPMC Université \\ Paris 6, CNRS, 75005 Paris, France; psellitto@Imd.ens.fr \\ 5 Remote Sensing Group, UK Research and Innovation, Science and Technology Facilities Council, Rutherford \\ Appleton Laboratory, Harwell, Oxford OX11 0QX, UK \\ 6 Istituto Nazionale di Geofisica e Vulcanologia, Osservatorio Etneo, I95123 Catania, Italy; \\ giuseppe.salerno@ingv.it \\ * Correspondence: a.mcgonigle@sheffield.ac.uk; Tel.: +44-114-222-7961
}

Received: 22 April 2018; Accepted: 24 April 2018; Published: 30 April 2018

Keywords: volcanic plumes; volcanic gases; volcanic geochemistry; atmospheric remote sensing; radiative forcing; atmospheric chemistry

Here we introduce a Special Issue of Geosciences focused on the scientific research field of 'Volcanic Plumes: Impacts on the atmosphere and insights into volcanic processes'. We would like to firstly thank those who have participated in this endeavour, in particular the authors who have chosen to submit their research outputs to this collection of articles, as well as the reviewers who have devoted their time, in a manner which has been invaluable in improving all the papers that appear here. This research theme provides a truly inter-system perspective on the dynamics of planet Earth, spanning the geosphere and the atmosphere and covering processes, which occur over a wide variety of timescales, and have significant impacts on human beings and the biosphere, not least via volcanogenic climate change. The title was deliberately chosen to encompass to the wide variety of scientific research occurring within this field and we are very pleased to see that the range of articles appearing here does span that of current enquiry regarding volcanic plumes.

Firstly, there are articles focused on the impacts on the atmosphere of volcanic plumes. In particular, Roberts [1] outlines an intriguing study into the rather recently discovered ozone destroying chemical processes, which occur within tropospheric volcanic plumes. By presenting results from measurements of ozone in the plume of a low halogen emitting volcano (Kîlauea), and combining these data with those from higher halogen emitters (Etna and Mt. Redoubt), as well as model simulations, new insights are offered into the role of halogen species in driving these chemical processes. On this theme, sun photometric observations of aerosol optical depth are also reported by Sellitto et al. [2], concerning the plume released from Pacaya volcano, Guatemala. Constraining the microphysics of aerosol particles in volcanic plumes is important to enable better understanding of the radiative properties of these emissions, and hence their potential role in modulation of radiative transfer and climatic dynamics. Another work, which is focused on measuring from the ground the properties of volcanic gas plumes is that of Pfeiffer et al. [3], who deployed a variety of spectroscopic and in-situ sampling tools, under challenging environmental conditions, to constrain the gas chemistry, emission rate and aerosol properties of the plume arising from the Bárðarbunga fissure eruption in Iceland. 
This eruption was the greatest in Iceland in the last 200 years and one of the most polluting volcanic events in centuries. Capture of data such as these is very important in terms of parameterising plume dispersal models, with a view to better mitigating the impacts on human beings of eruption clouds.

The second major focus of the volume title is on the use of plume data to inform our understanding of the subterranean processes, which drive volcanic activity at the surface. Here two articles appeared, the first of which was by Terray et al. [4]. In this paper, the authors report on the disequilibria between the radioactive species: ${ }^{210} \mathrm{~Pb},{ }^{210} \mathrm{Bi}$ and ${ }^{210} \mathrm{Po}$ in the plume of Mt. Etna. Such data have been used for some decades in attempts to constrain underground magma dynamics and degassing kinetics. Here a novel basaltic degassing model is put forward, based on a Monte-Carlo simulation, which the authors argue delivers a better fit to the observed data than those adopted previously. Linkage between models and observed gas data is also the theme of the next article, concerning magmatic-degassing dynamics, by Pering and McGonigle [5]. Here the authors use high time resolution remotely sensed degassing data (from ultraviolet cameras; see below) in tandem with mathematical models to provide an overarching model classification for puffing and strombolian degassing modes in basaltic volcanism for the first time.

Beyond the above topics, there were a number of articles, which focused on the development of hardware and software/algorithmic protocols for remotely sensing the properties of volcanic plumes. Within this realm there were three pieces focused on aerial/satellite observation platforms, and a further four concerning ground based configurations. In terms of the first approach, which is relevant for aviation in the event of large eruptions, or constraining the climatic impacts of eruptions, there was a contribution from Guermazi et al. [6] regarding thermal infrared methodologies. In particular, the authors focus on better constraining the concurrent impact of both sulphur dioxide and secondary sulphate aerosols on the signals received in the sensors' measurement bands. This radiative interference between sulphur dioxide and secondary sulfate aerosols has not been investigated before, hence this study paves the way towards more precise quantitative observation of these components of volcanic plumes. In addition, Licciardi et al. [7] report on a study concerning hyperspectral imaging in volcanology, aimed at unmixing the relative spectral effects of the ground covering and plume composition on the radiation signal received by the sensor. Here a nonlinear approach, based on machine learning, is adopted, which is in contrast to the linear techniques applied hitherto with a view to better resolving these relative effects, and it hence informs a more robust model interpretation of captured volcano-hyperspectral data. Furthermore, Corradini et al. [8] attempted a down-scaling of satellite-based observations to characterise proximal parameters of the volcanic activity, such as the start date and duration of eruptions, plume discharge rates and plume heights. This is of particular relevance to the anticipated increased future use of space-based platforms in volcanic monitoring.

Finally, there are a number of pieces focused on the ground based remote sensing techniques applied to volcanic plume sensing, an approach of particular importance in volcano monitoring, where high time resolution is helpful and the pre-eruptive plumes are often rather too weak to be resolved from space. An excellent overview of this domain is provided by Platt et al. [9], who review the significant development of this field in recent decades, which has led to a large number of volcanoes, spanning the globe, now being the focus of routine remotely sensed gas observations, enabling the observer to remain stationed at a safe distance from the source. This article provides an overview of a range of imaging and spectroscopic approaches, which have been applied in this arena. McGonigle et al. [10] focus on a particular modality within this genre: ultraviolet imaging of volcanic plumes, which has emerged over the last decade to provide plume emission rate data with unprecedented time resolutions of order $1 \mathrm{~s}$. The associated hardware and software protocols are covered as well as the significant novel scientific possibilities that this approach now enables, in particular the capacity to relate high time resolution gas flux data with volcano geophysical data for the first time to bridge between two previously rather separate branches of volcanology.

Gliß et al. [11] push the theme of UV imaging further by reporting on open access Python code aimed at handling all the processing steps, which are required to generate volcanic sulphur 
dioxide gas fluxes from raw acquired camera data. In particular, this provides calibration, plume speed determination and light dilution correction functionality, with the aim of expediting the wider uptake of this methodology amongst the international volcanology community. The final article, by Santoro et al. [12] concerns active remote sensing of carbon dioxide emissions from Mt. Etna using a LiDAR based system. Increases in the emission of this species can be a signature of forthcoming volcanic eruptions, hence this approach has very great potential in hazard assessment. The technique can also be applied at considerable distances from the source, providing significant safety benefits relative to the traditionally applied proximal measurements of this species at/near active vents.

It seems an apposite moment to publish this Special Issue, given the very significant developments, which have occurred in the area of volcanic plumes in the last decade or so. It is also hoped that the wide scientific coverage of the articles presented here will provide the reader with a good overview of the state of the art across the breadth of this field. These articles additionally pave the way for the exciting developments that are likely to follow in the following decade based on anticipated improvements in volcano monitoring instrumentation, deployed from both ground and space based platforms, in addition to improved data analytic procedures. With additional improvements in models concerning both underground gas behaviour as well as the physical dispersal and chemical and microphysical evolution of plumes in the atmosphere, we look forward to the further development of this field in the decade to come.

Author Contributions: The authors contributed equally to the writing of this Editorial.

Conflicts of Interest: The authors declare no conflict of interest.

\section{References}

1. Roberts, T.J. Ozone Depletion in Tropospheric Volcanic Plumes: From Halogen-Poor to Halogen-Rich Emissions. Geosciences 2018, 8, 68. [CrossRef]

2. Sellitto, P.; Spampinato, L.; Salerno, G.G.; La Spina, A. Aerosol Optical Properties of Pacaya Volcano Plume Measured with a Portable Sun-Photometer. Geosciences 2018, 8, 36. [CrossRef]

3. Pfeffer, M.A.; Bergsson, B.; Barsotti, S.; Stefánsdóttir, G.; Galle, B.; Arellano, S.; Conde, V.; Donovan, A.; Ilyinskaya, E.; Burton, M.; et al. Ground-Based Measurements of the 2014-2015 Holuhraun Volcanic Cloud (Iceland). Geosciences 2018, 8, 29. [CrossRef]

4. Terray, L.; Gauthier, P.-J.; Salerno, G.; Caltabiano, T.; La Spina, A.; Sellitto, P.; Briole, P. A New Degassing Model to Infer Magma Dynamics from Radioactive Disequilibria in Volcanic Plumes. Geosciences 2018, 8, 27. [CrossRef]

5. Pering, T.D.; McGonigle, A.J.S. Combining Spherical-Cap and Taylor Bubble Fluid Dynamics with Plume Measurements to Characterize Basaltic Degassing. Geosciences 2018, 8, 42. [CrossRef]

6. Guermazi, H.; Sellitto, P.; Serbaji, M.M.; Legras, B.; Rekhiss, F. Assessment of the Combined Sensitivity of Nadir TIR Satellite Observations to Volcanic $\mathrm{SO}_{2}$ and Sulphate Aerosols after a Moderate Stratospheric Eruption. Geosciences 2017, 7, 84. [CrossRef]

7. Licciardi, G.A.; Sellitto, P.; Piscini, A.; Chanussot, J. Nonlinear Spectral Unmixing for the Characterisation of Volcanic Surface Deposit and Airborne Plumes from Remote Sensing Imagery. Geosciences 2017, 7, 46. [CrossRef]

8. Corradini, S.; Guerrieri, L.; Lombardo, V.; Merucci, L.; Musacchio, M.; Prestifilippo, M.; Scollo, S.; Silvestri, M.; Spata, G.; Stelitano, D. Proximal Monitoring of the 2011-2015 Etna Lava Fountains Using MSG-SEVIRI Data. Geosciences 2018, 8, 140. [CrossRef]

9. Platt, U.; Bobrowski, N.; Butz, A. Ground-Based Remote Sensing and Imaging of Volcanic Gases and Quantitative Determination of Multi-Species Emission Fluxes. Geosciences 2018, 8, 44. [CrossRef]

10. McGonigle, A.J.S.; Pering, T.D.; Wilkes, T.C.; Tamburello, G.; D'Aleo, R.; Bitetto, M.; Aiuppa, A.; Willmott, J.R. Ultraviolet Imaging of Volcanic Plumes: A New Paradigm in Volcanology. Geosciences 2017, 7, 68. [CrossRef] 
11. Gliß, J.; Stebel, K.; Kylling, A.; Dinger, A.S.; Sihler, H.; Sudbø, A. Pyplis-A Python Software Toolbox for the Analysis of $\mathrm{SO}_{2}$ Camera Images for Emission Rate Retrievals from Point Sources. Geosciences 2017, 7, 134. [CrossRef]

12. Santoro, S.; Parracino, S.; Fiorani, L.; D'Aleo, R.; Di Ferdinando, E.; Giudice, G.; Maio, G.; Nuvoli, M.; Aiuppa, A. Volcanic Plume $\mathrm{CO}_{2}$ Flux Measurements at Mount Etna by Mobile Differential Absorption Lidar. Geosciences 2017, 7, 9. [CrossRef] 